\title{
The Clinicopathological Features of BRAF Mutated Papillary Thyroid Cancers in Chinese Patients
}

\author{
Li-Bo Yang, ${ }^{1,2}$ Lin-Yong Sun, ${ }^{1}$ Yong Jiang, ${ }^{1}$ Ying Tang, ${ }^{1}$ Zhi-Hui Li, ${ }^{3}$ Hong-Ying Zhang, \\ Hong $\mathrm{Bu}^{1,2}$ and Feng Ye $\mathrm{C}^{1,2}$ \\ ${ }^{1}$ Department of Pathology, West China Hospital, Sichuan University, Chengdu 610041, China \\ ${ }^{2}$ Laboratory of Pathology, West China Hospital, Sichuan University, Chengdu 610041, China \\ ${ }^{3}$ Department of Thyroid and Breast Surgery, West China Hospital, Sichuan University, Chengdu 610041, China \\ Correspondence should be addressed to Feng Ye; fengye@scu.edu.cn
}

Received 20 May 2015; Revised 13 July 2015; Accepted 14 July 2015

Academic Editor: Diego Russo

Copyright (C) 2015 Li-Bo Yang et al. This is an open access article distributed under the Creative Commons Attribution License, which permits unrestricted use, distribution, and reproduction in any medium, provided the original work is properly cited.

\begin{abstract}
The BRAF ${ }^{\mathrm{V} 600 \mathrm{E}}$ mutation is commonly found in papillary thyroid cancers (PTCs) at different frequencies in different regions. However, the association between the BRAF ${ }^{\mathrm{V} 600 \mathrm{E}}$ mutation and clinicopathological features in Chinese PTC patients is unknown. A total of 543 Chinese patients with histologically confirmed PTC were enrolled in this study. For the BRAF mutation assay, the target fragments were amplified and sequenced with an ABI 3500 gene analyzer. In 170 of 543 samples (31.3\%), the BRAF ${ }^{\mathrm{V} 600 \mathrm{E}}$ mutation was detected. In the bivariate analysis, the $\mathrm{BRAF}^{\mathrm{V} 600 \mathrm{E}}$ mutation showed an association with bilaterality, tumor size, extrathyroidal invasion, and lymph node metastases (LNM). However, in the multivariate analysis, the BRAF ${ }^{\mathrm{V} 600 \mathrm{E}}$ mutation was positively related to only tumor size $(>1 \mathrm{~cm})$ and extrathyroidal invasion. In addition, the multivariate analysis also showed that the age at diagnosis $(<45 \mathrm{y})$ and tumor size $(>1 \mathrm{~cm})$ were independent predictors for LNM. In this study, the BRAF ${ }^{\mathrm{V} 600 \mathrm{E}}$ mutation is positively associated with worse prognostic factors, including larger tumor size and the tumor extending to the thyroid capsule or extrathyroidal region; however, it is not an independent predictor for LNM.
\end{abstract}

\section{Introduction}

The incidence of thyroid cancer has increased over the past few decades in different countries [1], including China [1,2]. Among all the types of thyroid cancers, the increased rate of papillary thyroid cancer (PTC) is particularly high [3]. PTC, which is the most common subtype of thyroid cancer, represents approximately $80 \%-90 \%$ of all thyroid cancers [35].

The BRAF ${ }^{\mathrm{V} 600 \mathrm{E}}$ mutation leads to uncontrolled activation of the MAPK pathway, which is crucial for both tumor initiation and the progression of PTC $[6,7]$. Gene mutations and/or chromosome changes in certain effectors of the MAPK pathway, such as BRAF mutations, RAS mutations, and RET/PTC rearrangements, lead to constitutive activation of cell signaling, resulting in uncontrolled proliferation and carcinogenesis. However, the association between $\mathrm{BRAF}^{\mathrm{V} 600 \mathrm{E}}$ mutation and clinicopathological features remains controversial. Several studies showed that the occurrence of the $\mathrm{BRAF}^{\mathrm{V} 600 \mathrm{E}}$ mutation in PTC was related to aggressive features, including extrathyroidal invasion and lymph node metastases [8-10]. However, other authors did not find this association [11, 12]. Lymph node metastasis (LNM) is an aggressive factor and is associated with recurrence and cancer-related mortality $[13,14]$. Patients who underwent central lymph node dissection suffered a greater risk of permanent hypoparathyroidism or permanent nerve injury [15]. Preoperative medical checkups did not identify all metastases, including some small metastases in the lymph node [16]. This common clinical dilemma is troubling, although some authors reported that the $\mathrm{BRAF}^{\mathrm{V} 600 \mathrm{E}}$ mutation is an independent predictor of central node metastasis [17-20]. Therefore, it is necessary to determine if the $\mathrm{BRAF}^{\mathrm{V} 600 \mathrm{E}}$ mutation is a predictor of LNM in Chinese PTC patients and 
if it may help surgeons decide whether to perform lymph node dissection.

In addition, the BRAF mutation may be involved in the decreased expression of iodine metabolism genes, such as the sodium/iodide symporter and TSH receptor [2124], leading to iodine resistance. Target inhibition of BRAF activity is currently the alternative therapeutic approach for iodine-refractory PTC $[25,26]$, and several small molecule inhibitors of BRAF have been developed, including selumetinib, sorafenib, BAY 43-9006, PLX4032, RAF265, and PLX4720 [26-29], each with different selectivities [30, 31]. Furthermore, some of these drugs are currently in phase II and phase III clinical trial studies for the treatment of thyroid cancer $[26,31]$. By contrast, the incidence of thyroid cancer is different depending on race and geographic regions. The countries with a high reported incidence of thyroid cancer are Polynesia, Iceland, Italy, Israel, Finland, Hong Kong, China, Canada, and United States; the highest rate is found in New Caledonia, which has reported an approximately 10fold higher rate than most developed countries [32-34]. The incidence of the $\mathrm{BRAF}^{\mathrm{V} 600 \mathrm{E}}$ mutation in PTC also has a wide range of frequencies, from 25 to $90 \%$ [8, 12, 35-38]. Jeong et al. [8] reported that the frequency of the BRAF mutation in Korean PTC patients was $90 \%$. Currently, this is the highest frequency of the BRAF mutation reported. The different geographic region is the most likely explanation for this phenomenon [39]. There is a clear need to determine the frequency of the $\mathrm{BRAF}^{\mathrm{V} 600 \mathrm{E}}$ mutation, prior to administering any targeted clinical therapies into Chinese PTC patients.

In this study, we investigated the status of the $\mathrm{BRAF}^{\mathrm{V} 600 \mathrm{E}}$ mutation in primary tumors from 543 Chinese PTC patients to identify correlations between this genetic event and clinicopathological factors.

\section{Materials and Methods}

2.1. Patients and Samples. This study was a retrospective study and all data were analyzed anonymously. The Institutional Ethics Committee of the West China Hospital approved this study. Patients with histologically confirmed PTC from January 2013 to December 2014 were assessed for this study. A total of 597 primary cases that underwent thyroidectomy and routine central lymph node dissection at the West China Hospital were selected for further study. Of these, 54 patients were excluded because of incomplete patient information or an inadequate tumor sample (Figure 1). Finally, we included 543 formalin-fixed paraffin-embedded (FFPE) PTC specimens in this study. Some patients selectively underwent lateral neck lymph node dissection if the preoperative medical checkups, such as ultrasound, computed tomography, fine needle aspiration cytology, or cervical lymph node biopsy, suggested metastatic papillary cancer. Demographic and clinicopathological features, including gender, age of the patient at diagnosis, multifocality, bilaterality, tumor size, extrathyroidal invasion, and lymph node status, were collected from the patient medical histories and pathology reports. Specifically, "extrathyroidal invasion" in this study indicates that the tumor invaded the thyroid capsule or grew into the extrathyroidal region. The International

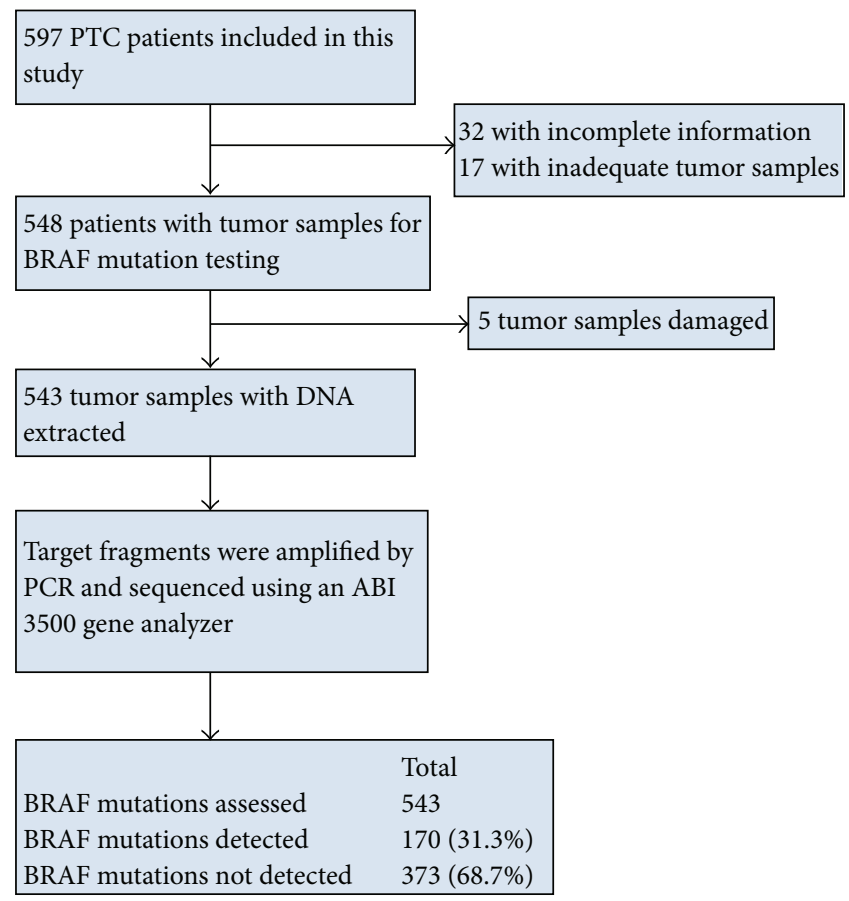

FIGURE 1: Flowchart showing the inclusion and results of the $\mathrm{BRAF}^{\mathrm{V} 600 \mathrm{E}}$ mutation in Chinese PTC patients.

Union against Cancer/American Joint Committee on Cancer (UICC/AJCC) tumor node metastasis (TNM) classification system was used for tumor staging [40]. All information regarding these samples is presented in Table 1.

2.2. DNA Extraction and BRAF Analysis. A total of 2-8 sections, each with a thickness of $5 \mu \mathrm{m}$ and a surface area of approximately $200 \mathrm{~mm}^{2}$ in total, were used for DNA extraction. A QIAamp DNA FFPE Tissue Kit (QIAGEN cat.56404) was used for genomic DNA extraction from the FFPE blocks. Briefly, the paraffin was removed with xylene and washed with ethanol. Air-dried tissue was resuspended in ATL buffer with proteinase $\mathrm{K}$ and incubated overnight. After a 1 hour incubation at $90^{\circ} \mathrm{C}, 200 \mu \mathrm{L}$ of $\mathrm{AL}$ was added to the sample. The entire mixture was loaded onto a QIAamp MinElute column. Then, the sample was washed with AW1 and AW2 wash buffer, and the genomic DNA was eluted in $50 \mu \mathrm{L}$ of ATE buffer. The polymerase chain reaction (PCR) primers used were the forward primer, $5^{\prime}$ TGCTTGCTCTGATAGGAAAATG- $3^{\prime}$, and reverse primer, $5^{\prime}$-AGCCTCAATTCTTACCATCCA- $3{ }^{\prime}$. The thermocycler program was set as follows: $94^{\circ} \mathrm{C} 3 \mathrm{~min}, 35 \times\left(94^{\circ} \mathrm{C} 30 \mathrm{sec}\right.$, $60^{\circ} \mathrm{C} 30 \mathrm{sec}$, and $72^{\circ} \mathrm{C} 30 \mathrm{sec}$ ), and $72^{\circ} \mathrm{C}$ for $5 \mathrm{~min}$. PCR products (191 bp) were subjected to automated sequencing using an ABI PRISM 3500 (Applied Biosystems, Foster City, CA, USA). All mutated cases were confirmed twice with independent PCR assays.

2.3. Statistical Analysis. Statistical analysis was performed with SPSS 16.0 (SPSS, Inc., Chicago, IL). Pearson $\chi^{2}$ test and Fisher's exact test were used to calculate the bivariate analysis 
TABLE 1: Clinicopathological features in the study of PTC in this study.

\begin{tabular}{lc}
\hline Characteristics & Number \\
\hline Number of patients & 543 \\
Gender & \\
Female & $409(75.3 \%)$ \\
$\quad$ Male & $134(24.7 \%)$ \\
Age at diagnosis, years & \\
$\quad$ Mean \pm SD & $42.10 \pm 12.12$ \\
Range & \\
$\quad<45$ & $219(40.3 \%)$ \\
$\quad \geq 45$ & $324(59.7 \%)$ \\
Multifocality & $230(42.4 \%)$ \\
Bilaterality & $125(23.0 \%)$ \\
Tumor size, cm & \\
$\quad$ Mean \pm SD & $1.14 \pm 0.93$ \\
Range & \\
$\quad \leq 1$ & $345(63.5 \%)$ \\
$\quad(1,2]$ & $147(27.1 \%)$ \\
$\quad(2,4]$ & $42(7.7 \%)$ \\
$\quad>4$ & $9(1.7 \%)$ \\
Extra-thyroidal invasion & $359(66.1 \%)$ \\
LNM & $321(59.1 \%)$ \\
Central LNM & $312(57.5 \%)$ \\
Lateral LNM & $123(22.7 \%)$ \\
BRAF ${ }^{V 600 E}$ mutation & $170(31.3 \%)$ \\
AJCC stage & \\
I & $434(79.9 \%)$ \\
II & $6(1.1 \%)$ \\
III & $70(12.9 \%)$ \\
\hline & $33(6.1 \%)$ \\
\hline
\end{tabular}

of the relationship between the $\mathrm{BRAF}^{\mathrm{V} 600 \mathrm{E}}$ mutation status and clinicopathological features, including gender, age of the patient at diagnosis, multifocality, bilaterality, tumor size, extrathyroidal invasion, lymph node status, and AJCC stage. A multivariate analysis was performed using binary logistic regression analysis for variables, which were significant in the bivariate analysis. Binary logistic regression analysis was also conducted to identify the variables associated with LNM independently. These variables were assessed using preoperative examination and intraoperative frozen-section examination, including gender, age of the patient at diagnosis, multifocality, bilaterality, tumor size, and extrathyroidal invasion. Continuous variables are presented as the mean \pm standard deviation. Independent samples $t$-tests were used for analysis. The two-sided significance level was set at $P<$ 0.05 .

\section{Results}

3.1. Baseline Clinicopathological Characteristics. The information for the 543 patients diagnosed with PTC included in this retrospective study is summarized in Table 1 . A total of
409 females $(75.3 \%)$ and 134 males $(24.7 \%)$ with a mean age of $42.10 \pm 12.12$ years at the time of diagnosis were included. The mean tumor size was $1.14 \pm 0.93 \mathrm{~cm}$. Bilateral PTC was present in 125 patients (23.0\%). Multifocal PTC was found in 230 patients $(42.4 \%)$. A total of 359 patients $(66.1 \%)$ had extrathyroidal invasion. LNM was discovered in 321 patients (59.1\%). Of all PTC patients, 170 patients (31.3\%) harbored BRAF $^{\mathrm{V} 600 \mathrm{E}}$. For tumor stage, 434 patients $(79.9 \%)$ were at stage I, 6 patients $(1.1 \%)$ were at stage II, 70 patients $(12.9 \%)$ were at stage III, and 33 patients $(6.1 \%)$ were at stage IV.

3.2. Association between BRAF $F^{V 600 E}$ Mutation and Clinicopathological Characteristics. Bivariate analysis showed that the tumor size, bilaterality, extrathyroidal invasion and LNM are associated with $\mathrm{BRAF}^{\mathrm{V600E}}$ (Table 2). There was no significant association of the $\mathrm{BRAF}^{\mathrm{V} 600 \mathrm{E}}$ mutation with gender, age at diagnosis, multifocality, and AJCC stage in our study. The relationship between $\mathrm{BRAF}^{\mathrm{V} 600 \mathrm{E}}$ and tumor size were confirmed by $t$-test. The mean tumor size of the 170 patients with $\mathrm{BRAF}^{\mathrm{V} 600 \mathrm{E}}$ was $1.47 \pm 0.99 \mathrm{~cm}$, whereas the mean tumor size of the wild type patients was $0.98 \pm 0.87 \mathrm{~cm}(t=5.80$, $P<0.001)$.

Additionally, multivariate analysis (binary logistic regression) showed that $\mathrm{BRAF}^{\mathrm{V} 600 \mathrm{E}}$ was positively associated only with tumor size $(>1 \mathrm{~cm})$ and extrathyroidal invasion (Table 3).

3.3. Predictive Factors of LNM. We then performed a binary logistic regression analysis to determine whether $\mathrm{BRAF}^{\mathrm{V} 600 \mathrm{E}}$ is an independent predictive factor for central LNM (Table 4) or lateral LNM (Table 5). After controlling for gender, age at diagnosis, multifocality, bilaterality, extrathyroidal invasion, tumor size, and $\mathrm{BRAF}^{\mathrm{V} 600 \mathrm{E}}$ mutation, we found that the $\mathrm{BRAF}^{\mathrm{V} 600 \mathrm{E}}$ mutation was not an independent predictor for central LNM or lateral LNM. However, age at diagnosis $(<45 \mathrm{y})$ and tumor size $(>1 \mathrm{~cm})$ were independent predictors for both central LNM and lateral LNM. Gender (female) was an independent predictor for only central LNM, whereas bilateral tumor and extrathyroidal invasion were independent predictors for only lateral LNM.

\section{Discussion}

Sanger sequencing, PCR, and immunohistochemistry are three primary methods for detecting the BRAF mutation. There were no significant differences among these methods [41]. However, PCR is commonly used because of its high-efficiency; therefore, we only used this method to detect mutations in this study. There are more than ten types of BRAF mutation variants reported for malignant tumors such as bladder, melanoma, and PTC. These variants include BRAF $^{\mathrm{V} 600 \mathrm{E}}, \mathrm{BRAF}^{\mathrm{V} 600 \mathrm{D}}, \mathrm{BRAF}^{\mathrm{V} 600 \mathrm{M}}, \mathrm{BRAF}^{\mathrm{V} 600 \mathrm{~V}}$, BRAF $^{\mathrm{V} 600 \mathrm{R}}, \mathrm{BRAF}^{\mathrm{V} 600 \mathrm{E} 2}, \mathrm{BRAF}^{\mathrm{V} 600 \mathrm{Q}}, \mathrm{BRAF}^{\mathrm{V} 600 \mathrm{~L}}$, and $\mathrm{BRAF}^{\mathrm{V} 600 \mathrm{~K}}$ [42-44]. However, in our study, we only identified the common type of BRAF mutation variant, BRAF ${ }^{\mathrm{V} 600 \mathrm{E}}$. Therefore, theoretically, any drugs that specifically inhibit B-type RAF kinase should at least target the mutant type 
TABLE 2: Relationship between the BRAF ${ }^{\mathrm{V} 600 \mathrm{E}}$ mutation and clinicopathological factors in PTC.

\begin{tabular}{|c|c|c|c|c|c|}
\hline & Total n $(\%)$ & $\mathrm{BRAF}^{\mathrm{V} 600 \mathrm{E}}$ & $n(\%)$ & $\gamma^{2}$ & $P$ value \\
\hline & IVtai, $n(0)$ & Mutation $(n=170)$ & Wild $(n=373)$ & & 1 value \\
\hline Gender & & & & 2.288 & 0.130 \\
\hline Female & $409(75.3)$ & $121(29.6)$ & $288(70.4)$ & & \\
\hline Male & $134(24.7)$ & $49(36.6)$ & $85(63.4)$ & & \\
\hline Age at diagnosis & & & & 0.234 & 0.629 \\
\hline$<45$ & $219(40.3)$ & $66(30.1)$ & $153(69.9)$ & & \\
\hline$\geq 45$ & $324(59.7)$ & $104(32.1)$ & $220(69.7)$ & & \\
\hline Multifocality & & & & 0.314 & 0.575 \\
\hline No & $313(57.6)$ & $95(30.4)$ & $218(69.6)$ & & \\
\hline Yes & $230(42.4)$ & $75(32.6)$ & $155(67.4)$ & & \\
\hline Bilaterality & & & & 4.703 & 0.030 \\
\hline No & $418(77.0)$ & $121(28.9)$ & $297(71.1)$ & & \\
\hline Yes & $125(23.0)$ & $49(39.2)$ & $76(60.8)$ & & \\
\hline Tumor size $(\mathrm{cm})$ & & & & 31.109 & $<0.001$ \\
\hline$\leq 1$ & $345(63.5)$ & $79(22.9)$ & $266(77.1)$ & & \\
\hline$>1$ & $198(36.5)$ & $91(46.0)$ & $107(54.0)$ & & \\
\hline Extrathyroidal invasion & & & & 23.142 & $<0.001$ \\
\hline No & $184(33.9)$ & $33(17.9)$ & $151(82.1)$ & & \\
\hline Yes & $359(66.1)$ & $137(38.2)$ & $222(61.8)$ & & \\
\hline LNM & & & & 7.452 & 0.006 \\
\hline No & $222(40.9)$ & $55(24.8)$ & $167(75.2)$ & & \\
\hline Yes & $321(59.1)$ & $115(35.8)$ & $206(64.2)$ & & \\
\hline AJCC stage & & & & 6.741 & 0.072 \\
\hline I & $434(79.9)$ & $132(30.4)$ & $302(69.6)$ & & \\
\hline II & $6(1.1)$ & $2(33.3)$ & $4(66.7)$ & & \\
\hline III & 70 (12.9) & $19(27.1)$ & $51(72.9)$ & & \\
\hline IV & $33(6.1)$ & $17(51.5)$ & $16(48.5)$ & & \\
\hline
\end{tabular}

TABLE 3: Multivariate analysis of the association between clinicopathological features and BRAF ${ }^{\mathrm{V} 600 \mathrm{E}}$ mutation.

\begin{tabular}{|c|c|c|c|c|}
\hline \multirow{2}{*}{ Features } & \multirow{2}{*}{ Odds ratio } & \multicolumn{2}{|c|}{ 95\% confidence interval } & \multirow{2}{*}{$P$ value } \\
\hline & & Lower bound & Upper bound & \\
\hline Bilaterality $(+)$ & 0.843 & 0.541 & 1.314 & 0.451 \\
\hline Extrathyroidal invasion $(+)$ & 2.284 & 1.458 & 3.576 & $<0.001$ \\
\hline Tumor size $(>1 \mathrm{~cm})$ & 2.319 & 1.548 & 3.473 & $<0.001$ \\
\hline $\operatorname{LNM}(+)$ & 1.172 & 0.774 & 1.774 & 0.454 \\
\hline
\end{tabular}

TABLE 4: Multivariate analysis of the association between clinicopathological features and central LNM.

\begin{tabular}{|c|c|c|c|c|}
\hline \multirow{2}{*}{ Features } & \multirow{2}{*}{ Odds ratio } & \multicolumn{2}{|c|}{$95 \%$ confidence interval } & \multirow{2}{*}{$P$ value } \\
\hline & & Lower bound & Upper bound & \\
\hline Gender (female) & 2.441 & 1.552 & 3.840 & $<0.001$ \\
\hline Age at diagnosis $(<45 y)$ & 2.661 & 1.818 & 3.894 & $<0.001$ \\
\hline Multifocality $(+)$ & 1.115 & 0.692 & 1.797 & 0.655 \\
\hline Bilaterality $(+)$ & 1.641 & 0.911 & 2.957 & 0.099 \\
\hline Extrathyroidal invasion $(+)$ & 1.389 & 0.932 & 2.072 & 0.107 \\
\hline Tumor size $(>1 \mathrm{~cm})$ & 3.288 & 2.157 & 5.010 & $<0.001$ \\
\hline $\mathrm{BRAF}^{\mathrm{V} 600 \mathrm{E}}$ mutation $(+)$ & 0.954 & 0.624 & 1.457 & 0.826 \\
\hline
\end{tabular}


TABLE 5: Multivariate analysis of the association between clinicopathological features and lateral LNM.

\begin{tabular}{|c|c|c|c|c|}
\hline \multirow{2}{*}{ Features } & \multirow{2}{*}{ Odds ratio } & \multicolumn{2}{|c|}{$95 \%$ confidence interval } & \multirow{2}{*}{$P$ value } \\
\hline & & Lower bound & Upper bound & \\
\hline Gender (female) & 1.677 & 0.991 & 2.840 & 0.054 \\
\hline Age at diagnosis $(<45 y)$ & 2.625 & 1.582 & 4.356 & $<0.001$ \\
\hline Multifocality $(+)$ & 1.240 & 0.652 & 2.361 & 0.512 \\
\hline Bilaterality $(+)$ & 2.343 & 1.180 & 4.653 & 0.015 \\
\hline Extrathyroidal invasion $(+)$ & 2.323 & 1.304 & 4.136 & 0.004 \\
\hline Tumor size $(>1 \mathrm{~cm})$ & 4.821 & 2.997 & 7.755 & $<0.001$ \\
\hline BRAF $^{\mathrm{V} 600 \mathrm{E}}$ mutation $(+)$ & 1.175 & 0.722 & 1.911 & 0.516 \\
\hline
\end{tabular}

TABLE 6: Clinicopathological features associated with BRAF mutation in other studies.

\begin{tabular}{lccl}
\hline Studies & Country & Numbers of PTC patients & Clinicopathological features associated with BRAF mutation \\
\hline Howell et al. [18] & USA & 156 & Central LNM \\
Lim et al. [45] & Korea & 3130 & Tumor size, extrathyroidal extension, and LNM \\
Kim et al. [46] & Korea & 547 & Male gender, tumor size, and extrathyroidal extension \\
Xing et al. [47] & USA & 190 & Extrathyroidal extension, thyroid capsule invasion, and LNM \\
Nakayama et al. [48] & Japan & 54 & Older age, extrathyroidal extension, and LNM \\
Fugazzola et al. [49] & Italy & 260 & Older age \\
\hline
\end{tabular}

to inhibit the MAPK signaling pathway. Several studies reported probability of using a BRAF mutation inhibitor in iodine-refractory PTC $[25,26]$. This requires additional large-scale prospective trail to determine the availability and safety in patients.

The frequency of $\mathrm{BRAF}^{\mathrm{V} 600 \mathrm{E}}$ in Chinese PTC patients is $31.3 \%(170 / 543)$. The rate for $\mathrm{BRAF}^{\mathrm{V} 600 \mathrm{E}}$ mutation is approximately the same as reported in other countries $[6,7$, 31]. We did not identify any other BRAF mutation variant types as described in other studies. This may occur in a racedependent manner. Furthermore, the sample size was not large enough to identify all reported BRAF genetic changes.

Additionally, we analyzed the BRAF ${ }^{\mathrm{V} 600 \mathrm{E}}$ mutation and its clinical and pathological characteristics. Some studies reported that the $\mathrm{BRAF}^{\mathrm{V} 600 \mathrm{E}}$ mutation is related to male gender, older age, tumor size, thyroid capsular invasion, extrathyroidal extension, and LNM (Table 6) [18, 45-49]. These features indicated a poor outcome in PTC patients [50-52]. In our study, the BRAF ${ }^{\mathrm{V} 600 \mathrm{E}}$ mutation had a relationship with the tumor size, bilaterality, extrathyroidal invasion, and LNM based on the bivariate analysis. Multivariate analysis showed that tumor size $(>1 \mathrm{~cm})$ and extrathyroidal invasion had significant positive associations with the $\mathrm{BRAF}^{\mathrm{V} 600 \mathrm{E}}$ mutation, after controlling for the tumor size, bilaterality, extrathyroidal invasion, and LNM. This indicates that patients with a larger tumor size $(>1 \mathrm{~cm})$ or tumors extending to the thyroid capsule and extrathyroidal region are more likely to have $\mathrm{BRAF}^{\mathrm{V} 600 \mathrm{E}}$ mutation. In this study, we regard thyroid capsular invasion and extrathyroidal extension as "extrathyroidal invasion" to reduce errors, because the manner by which to distinguish extrathyroidal extension remains controversial. Although the publishers of the UICC/AJCC 7th edition TNM classification system have taken the degree of extrathyroidal extension into consideration, the criteria for defining extrathyroid extension are subjective and problematic because of the discontinuous capsule of the thyroid gland [53].

Several studies further defined the relationship between the BRAF mutation and the aggressiveness of thyroid tumor cells. Epithelial mesenchymal transition (EMT) is common in PTC invasion and is associated with LNM [54]. The BRAF mutation may render thyroid cells susceptible to transforming growth factor beta-induced EMT [55]. The aberrant methylation of tumor suppressor genes, leading to the increased aggressiveness of thyroid tumor cells, is also related to the BRAF mutation [56]. These studies help us understand the importance of this type of mutation in LNM. Several authors determined whether the BRAF ${ }^{\mathrm{V} 600 \mathrm{E}}$ mutation is a predictive factor for LNM to help surgeons decide which PTC patients should have lymph node dissection [17-19]. In our study, we did not find that the BRAF ${ }^{\mathrm{V} 600 \mathrm{E}}$ mutation was an independent predictor for LNM in Chinese PTC patients. However, the binary logistic regression analysis revealed that being female, younger age at diagnosis $(<45 \mathrm{y})$, and tumor size $>1 \mathrm{~cm}$ had a more significant association with central LNM. For lateral LNM, younger age at diagnosis $(<45 \mathrm{y})$, bilateral tumor, extrathyroidal invasion, and larger tumor size $(>1 \mathrm{~cm})$ were related. Therefore, Chinese PTC patients who are young $(<45 \mathrm{y})$ and have a larger tumor size $(>1 \mathrm{~cm})$ tend to have both central LNM and lateral LNM.

\section{Conclusion}

Our study shows that the occurrence of the $\mathrm{BRAF}^{\mathrm{V} 600 \mathrm{E}}$ mutation in Chinese patients is approximately the same as the other countries. The $\mathrm{BRAF}^{\mathrm{V} 600 \mathrm{E}}$ mutation is positively associated with worse prognostic factors, including larger 
tumor size and tumors extending to the thyroid capsule or extrathyroidal region. However, in our study, the $\mathrm{BRAF}^{\mathrm{V} 600 \mathrm{E}}$ mutation does not show predictive value for LNM.

\section{Conflict of Interests}

The authors declare that there is no conflict of interests regarding the publication of this paper.

\section{Authors' Contribution}

Li-Bo Yang and Lin-Yong Sun contributed equally to this work.

\section{Acknowledgments}

This work was funded by grants from the National Natural Science Foundation of China (31000601 and 81200461) and Young Investigator Scholar in Sichuan University (2012SCU04A14).

\section{References}

[1] C. La Vecchia, M. Malvezzi, C. Bosetti et al., "Thyroid cancer mortality and incidence: a global overview," International Journal of Cancer, vol. 136, no. 9, pp. 2187-2195, 2015.

[2] Y.-Q. Liu, S.-Q. Zhang, W.-Q. Chen et al., "Trend of incidence and mortality on thyroid cancer in China during 2003-2007," Zhonghua Liu Xing Bing Xue Za Zhi, vol. 33, no. 10, pp. 10441048, 2012.

[3] L. Davies and H. G. Welch, "Increasing incidence of thyroid cancer in the United States, 1973-2002," The Journal of the American Medical Association, vol. 295, no. 18, pp. 2164-2167, 2006.

[4] L. Leenhardt, P. Grosclaude, and L. Chérié-Challine, "Increased incidence of thyroid carcinoma in france: a true epidemic or thyroid nodule management effects? Report from the french thyroid cancer committee," Thyroid, vol. 14, no. 12, pp. 10561060, 2004.

[5] S. A. Hundahl, I. D. Fleming, A. M. Fremgen, and H. R. Menck, "A National Cancer Data Base report on 53,856 cases of thyroid carcinoma treated in the U.S., 1985-1995," Cancer, vol. 83, no. 12, pp. 2638-2648, 1998.

[6] Y. E. Nikiforov, "Molecular analysis of thyroid tumors," Modern Pathology, vol. 24, supplement 2, pp. S34-S43, 2011.

[7] M. Xing, "BRAF mutation in thyroid cancer," Endocrine-Related Cancer, vol. 12, no. 2, pp. 245-262, 2005.

[8] D. Jeong, Y. Jeong, J. H. Park et al., " $B R A F^{\mathrm{V} 600 \mathrm{E}}$ mutation analysis in papillary thyroid carcinomas by peptide nucleic acid clamp real-time PCR," Annals of Surgical Oncology, vol. 20, no. 3, pp. 759-766, 2013.

[9] X. Zheng, T. Xia, L. Lin et al., "BRAF ${ }^{\mathrm{V} 600 \mathrm{E}}$ status and clinical characteristics in solitary and multiple papillary thyroid carcinoma: experience of 512 cases at a clinical center in China," World Journal of Surgical Oncology, vol. 10, article 104, 2012.

[10] K.-L. Lin, O.-C. Wang, X.-H. Zhang, X.-X. Dai, X.-Q. Hu, and J.-M. Qu, "The BRAF mutation is predictive of aggressive clinicopathological characteristics in papillary thyroid microcarcinoma," Annals of Surgical Oncology, vol. 17, no. 12, pp. 32943300, 2010.
[11] Y.-S. Kim, J.-S. Kim, J.-S. Bae, and W.-C. Park, "Clinical implication of the BRAF ${ }^{\mathrm{V} 600 \mathrm{E}}$ mutation in papillary thyroid carcinoma," World Journal of Surgical Oncology, vol. 11, article 99, 2013.

[12] I. Yasuhiro, H. Yoshida, R. Maruo et al., "BRAF mutation in papillary thyroid carcinoma in a Japanese population: its lack of correlation with high-risk clinicopathological features and disease-free survival of patients," Endocrine Journal, vol. 56, no. 1, pp. 89-97, 2009.

[13] A. Bozec, O. Dassonville, E. Chamorey et al., "Clinical impact of cervical lymph node involvement and central neck dissection in patients with papillary thyroid carcinoma: a retrospective analysis of 368 cases," European Archives of Oto-Rhino-Laryngology, vol. 268, no. 8, pp. 1205-1212, 2011.

[14] Y. D. Podnos, D. Smith, L. D. Wagman, and J. D. I. Ellenhorn, "The implication of lymph node metastasis on survival in patients with well-differentiated thyroid cancer," American Surgeon, vol. 71, no. 9, pp. 731-734, 2005.

[15] S. Scherl, S. Mehra, J. Clain et al., "The effect of surgeon experience on the detection of metastatic lymph nodes in the central compartment and the pathologic features of clinically unapparent metastatic lymph nodes: what are we missing when we don't perform a prophylactic dissection of central compartment lymph nodes in papillary thyroid cancer?" Thyroid, vol. 24, no. 8, pp. 1282-1288, 2014.

[16] K. Shimamoto, H. Satake, A. Sawaki, T. Ishigaki, H. Funahashi, and T. Imai, "Preoperative staging of thyroid papillary carcinoma with ultrasonography," European Journal of Radiology, vol. 29, no. 1, pp. 4-10, 1998.

[17] C. Li, P. Aragon Han, K. C. Lee et al., "Does BRAF V600E mutation predict aggressive features in papillary thyroid cancer? results from four endocrine surgery centers," Journal of Clinical Endocrinology and Metabolism, vol. 98, no. 9, pp. 3702-3712, 2013.

[18] G. M. Howell, M. N. Nikiforova, S. E. Carty et al., "BRAF V600E mutation independently predicts central compartment lymph node metastasis in patients with papillary thyroid cancer," Annals of Surgical Oncology, vol. 20, no. 1, pp. 47-52, 2013.

[19] K. C. Lee, C. Li, E. B. Schneider et al., "Is BRAF mutation associated with lymph node metastasis in patients with papillary thyroid cancer?” Surgery, vol. 152, no. 6, pp. 977-983, 2012.

[20] J.-Y. Joo, J.-Y. Park, Y.-H. Yoon et al., "Prediction of occult central lymph node metastasis in papillary thyroid carcinoma by preoperative BRAF analysis using fine-needle aspiration biopsy: a prospective study," The Journal of Clinical Endocrinology \& Metabolism, vol. 97, no. 11, pp. 3996-4003, 2012.

[21] G. Riesco-Eizaguirre, I. Rodríguez, A. De la Vieja et al., “The $\mathrm{BRAF}^{\mathrm{V} 600 \mathrm{E}}$ oncogene induces transforming growth factor $\beta$ secretion leading to sodium iodide symporter repression and increased malignancy in thyroid cancer," Cancer Research, vol. 69 , no. 21, pp. 8317-8325, 2009.

[22] G. Oler and J. M. Cerutti, "High prevalence of BRAF mutation in a Brazilian cohort of patients with sporadic papillary thyroid carcinomas: correlation with more aggressive phenotype and decreased expression of iodide-metabolizing genes," Cancer, vol. 115, no. 5, pp. 972-980, 2009.

[23] C. Durante, E. Puxeddu, E. Ferretti et al., "Brief report: BRAF mutations in papillary thyroid carcinomas inhibit genes involved in iodine metabolism," The Journal of Clinical Endocrinology \& Metabolism, vol. 92, no. 7, pp. 2840-2843, 2007.

[24] D. Russo, G. Damante, E. Puxeddu, C. Durante, and S. Filetti, "Epigenetics of thyroid cancer and novel therapeutic targets," 
Journal of Molecular Endocrinology, vol. 46, no. 3, pp. R73-R81, 2011.

[25] D. A. Kleiman, D. Buitrago, M. J. Crowley et al., “Thyroid stimulating hormone increases iodine uptake by thyroid cancer cells during BRAF silencing," Journal of Surgical Research, vol. 182, no. 1, pp. 85-93, 2013.

[26] D. N. Hayes, A. S. Lucas, T. Tanvetyanon et al., "Phase II efficacy and pharmacogenomic study of selumetinib (AZD6244; ARRY142886) in iodine-131 refractory papillary thyroid carcinoma with or without follicular elements," Clinical Cancer Research, vol. 18, no. 7, pp. 2056-2065, 2012.

[27] C. Nucera, M. A. Nehs, S. S. Nagarkatti et al., "Targeting BRAFV600E with PLX4720 displays potent antimigratory and Anti-invasive activity in preclinical models of human thyroid cancer," Oncologist, vol. 16, no. 3, pp. 296-309, 2011.

[28] P. Hersey, L. Bastholt, V. Chiarion-Sileni et al., "Small molecules and targeted therapies in distant metastatic disease," Annals of Oncology, vol. 20, no. 4, pp. vi35-vi40, 2009.

[29] S. M. Wilhelm, C. Carter, L. Tang et al., "BAY 43-9006 exhibits broad spectrum oral antitumor activity and targets the RAF/MEK/ERK pathway and receptor tyrosine kinases involved in tumor progression and angiogenesis," Cancer Research, vol. 64, no. 19, pp. 7099-7109, 2004.

[30] E. Grande, J. J. Díez, C. Zafon, and J. Capdevila, “Thyroid cancer: molecular aspects and new therapeutic strategies," Journal of Thyroid Research, vol. 2012, Article ID 847108, 10 pages, 2012.

[31] Y. E. Nikiforov, "Thyroid carcinoma: molecular pathways and therapeutic targets," Modern Pathology, vol. 21, supplement 2, pp. S37-S43, 2008.

[32] T. Truong, Y. Rougier, D. Dubourdieu et al., "Time trends and geographic variations for thyroid cancer in New Caledonia, a very high incidence area (1985-1999)," European Journal of Cancer Prevention, vol. 16, no. 1, pp. 62-70, 2007.

[33] J. Albores-Saavedra, D. E. Henson, E. Glazer, and A. M. Schwartz, "Changing patterns in the incidence and survival of thyroid cancer with follicular phenotype-papillary, follicular, and anaplastic: a morphological and epidemiological study," Endocrine Pathology, vol. 18, no. 1, pp. 1-7, 2007.

[34] A. Lubina, O. Cohen, M. Barchana et al., "Time trends of incidence rates of thyroid cancer in Israel: what might explain the sharp increase," Thyroid, vol. 16, no. 10, pp. 1033-1040, 2006.

[35] B. Stanojevic, R. Dzodic, V. Saenko et al., "Mutational and clinico-pathological analysis of papillary thyroid carcinoma in Serbia," Endocrine Journal, vol. 58, no. 5, pp. 381-393, 2011.

[36] H.-Q. Guo, H. Zhao, Z.-H. Zhang, Y.-L. Zhu, T. Xiao, and Q.-J. Pan, "Impact of molecular testing in the diagnosis of thyroid fine needle aspiration cytology: data from Mainland China," Disease Markers, vol. 2014, Article ID 912182, 6 pages, 2014.

[37] M. N. Nikiforova, E. T. Kimura, M. Gandhi et al., "BRAF mutations in thyroid tumors are restricted to papillary carcinomas and anaplastic or poorly differentiated carcinomas arising from papillary carcinomas," Journal of Clinical Endocrinology and Metabolism, vol. 88, no. 11, pp. 5399-5404, 2003.

[38] H. Namba, M. Nakashima, T. Hayashi et al., "Clinical implication of hot spot BRAF mutation, V599E, in papillary thyroid cancers," Journal of Clinical Endocrinology and Metabolism, vol. 88, no. 9, pp. 4393-4397, 2003.

[39] N. Kurtulmus, M. Duren, U. Ince et al., "BRAF ${ }^{\mathrm{V} 600 \mathrm{E}}$ mutation in Turkish patients with papillary thyroid cancer: strong correlation with indicators of tumor aggressiveness," Endocrine, vol. 42, no. 2, pp. 404-410, 2012.
[40] S. B. B. D. Edge, C. C. Compton, A. G. Fritz, F. L. Greene, and A. Trotti, AJCC Cancer Staging Manual, Springer, New York, NY, USA, 7th edition, 2010.

[41] T. Qiu, H. Lu, L. Guo et al., "Detection of BRAF mutation in Chinese tumor patients using a highly sensitive antibody immunohistochemistry assay," Scientific Reports, vol. 5, article 9211, 2015.

[42] R. Kansal, L. Quintanilla-Martinez, V. Datta, J. Lopategui, G. Garshfield, and B. N. Nathwani, "Identification of the V600D mutation in Exon 15 of the BRAF oncogene in congenital, benign langerhans cell histiocytosis," Genes Chromosomes and Cancer, vol. 52, no. 1, pp. 99-106, 2013.

[43] C. Mao, J. Zhou, Z. Yang et al., "KRAS, BRAF and PIK3CA mutations and the loss of PTEN expression in Chinese patients with colorectal cancer," PLoS ONE, vol. 7, no. 5, Article ID e36653, 2012.

[44] C. M. Lovly, K. B. Dahlman, L. E. Fohn et al., "Routine multiplex mutational profiling of melanomas enables enrollment in genotype-driven therapeutic trials," PLoS ONE, vol. 7, no. 4, Article ID e35309, 2012.

[45] J. Y. Lim, S. W. Hong, Y. S. Lee et al., "Clinicopathologic implications of the BRAF ${ }^{\mathrm{V} 600 \mathrm{E}}$ mutation in papillary thyroid cancer: a subgroup analysis of 3130 cases in a single center," Thyroid, vol. 23, no. 11, pp. 1423-1430, 2013.

[46] S.-J. Kim, K. E. Lee, J. P. Myong et al., "BRAF" ${ }^{\mathrm{V} 600 \mathrm{E}}$ mutation is associated with tumor aggressiveness in papillary thyroid cancer," World Journal of Surgery, vol. 36, no. 2, pp. 310-317, 2012.

[47] M. Xing, D. Clark, H. Guan et al., "BRAF mutation testing of thyroid fine-needle aspiration biopsy specimens for preoperative risk stratification in papillary thyroid cancer," Journal of Clinical Oncology, vol. 27, no. 18, pp. 2977-2982, 2009.

[48] H. Nakayama, A. Yoshida, Y. Nakamura et al., "Clinical significance of BRAF (V600E) mutation and Ki-67 labeling index in papillary thyroid carcinomas," Anticancer Research, vol. 27, no. 5, pp. 3645-3649, 2007.

[49] L. Fugazzola, E. Puxeddu, N. Avenia et al., "Correlation between B-RAFV600E mutation and clinico-pathologic parameters in papillary thyroid carcinoma: data from a multicentric Italian study and review of the literature," Endocrine-Related Cancer, vol. 13, no. 2, pp. 455-464, 2006.

[50] S. Turanli, "Is the type of dissection in lateral neck metastasis for differentiated thyroid carcinoma important?" OtolaryngologyHead and Neck Surgery, vol. 136, no. 6, pp. 957-960, 2007.

[51] C. Durante, N. Haddy, E. Baudin et al., "Long-term outcome of 444 patients with distant metastases from papillary and follicular thyroid carcinoma: benefits and limits of radioiodine therapy," Journal of Clinical Endocrinology and Metabolism, vol. 91, no. 8, pp. 2892-2899, 2006.

[52] E. L. Mazzaferri and S. M. Jhiang, "Long-term impact of initial surgical and medical therapy on papillary and follicular thyroid cancer," The American Journal of Medicine, vol. 97, no. 5, pp. 418428, 1994.

[53] O. Mete, L. Rotstein, and S. L. Asa, "Controversies in thyroid pathology: thyroid capsule invasion and extrathyroidal extension," Annals of Surgical Oncology, vol. 17, no. 2, pp. 386-391, 2010.

[54] V. Vasko, A. V. Espinosa, W. Scouten et al., "Gene expression and functional evidence of epithelial-to-mesenchymal transition in papillary thyroid carcinoma invasion," Proceedings of the National Academy of Sciences of the United States of America, vol. 104, no. 8, pp. 2803-2808, 2007. 
[55] J. A. Knauf, M. A. Sartor, M. Medvedovic et al., "Progression of BRAF-induced thyroid cancer is associated with epithelialmesenchymal transition requiring concomitant MAP kinase and TGFB signaling," Oncogene, vol. 30, no. 28, pp. 3153-3162, 2011.

[56] S. Hu, D. Liu, R. P. Tufano et al., "Association of aberrant methylation of tumor suppressor genes with tumor aggressiveness and BRAF mutation in papillary thyroid cancer," International Journal of Cancer, vol. 119, no. 10, pp. 2322-2329, 2006. 


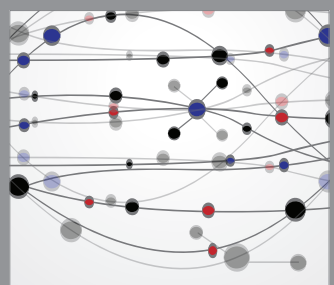

The Scientific World Journal
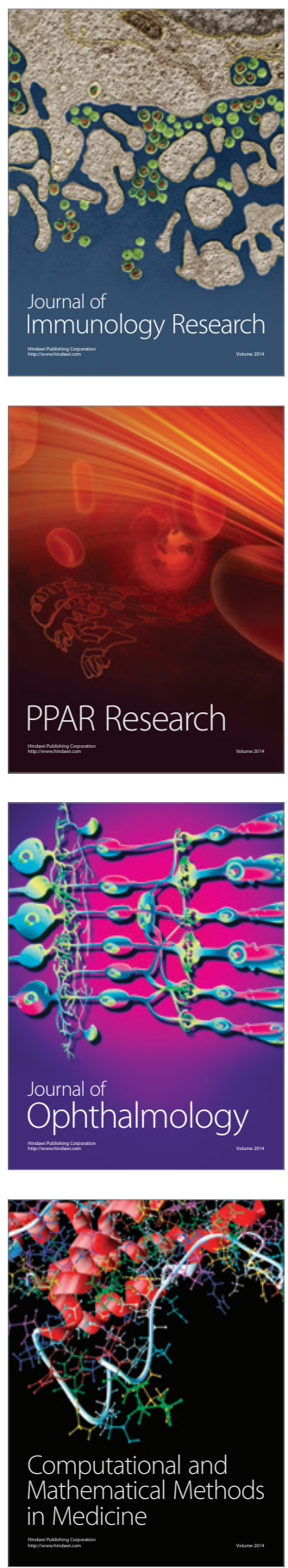

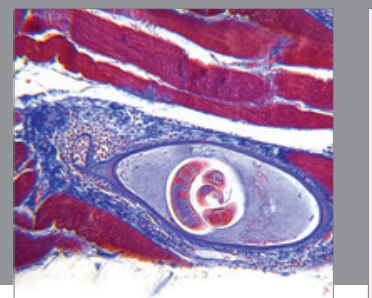

Gastroenterology

Research and Practice
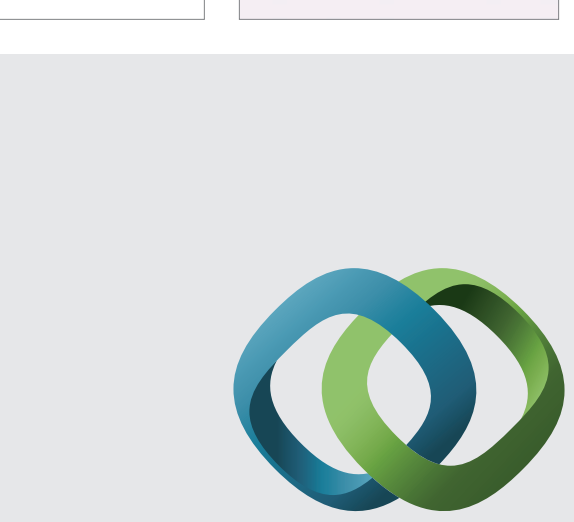

\section{Hindawi}

Submit your manuscripts at

http://www.hindawi.com
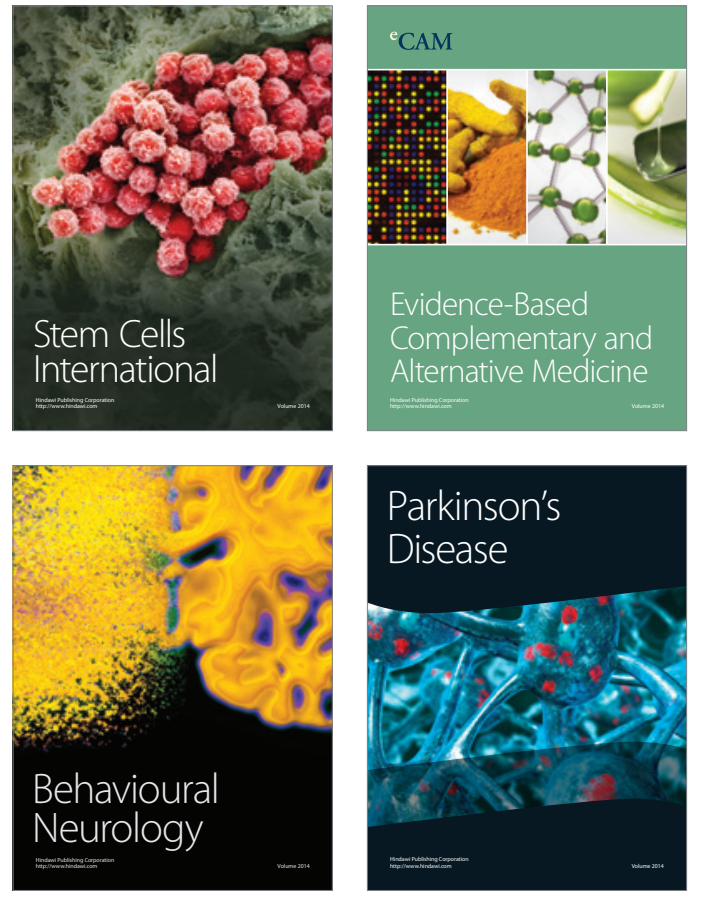
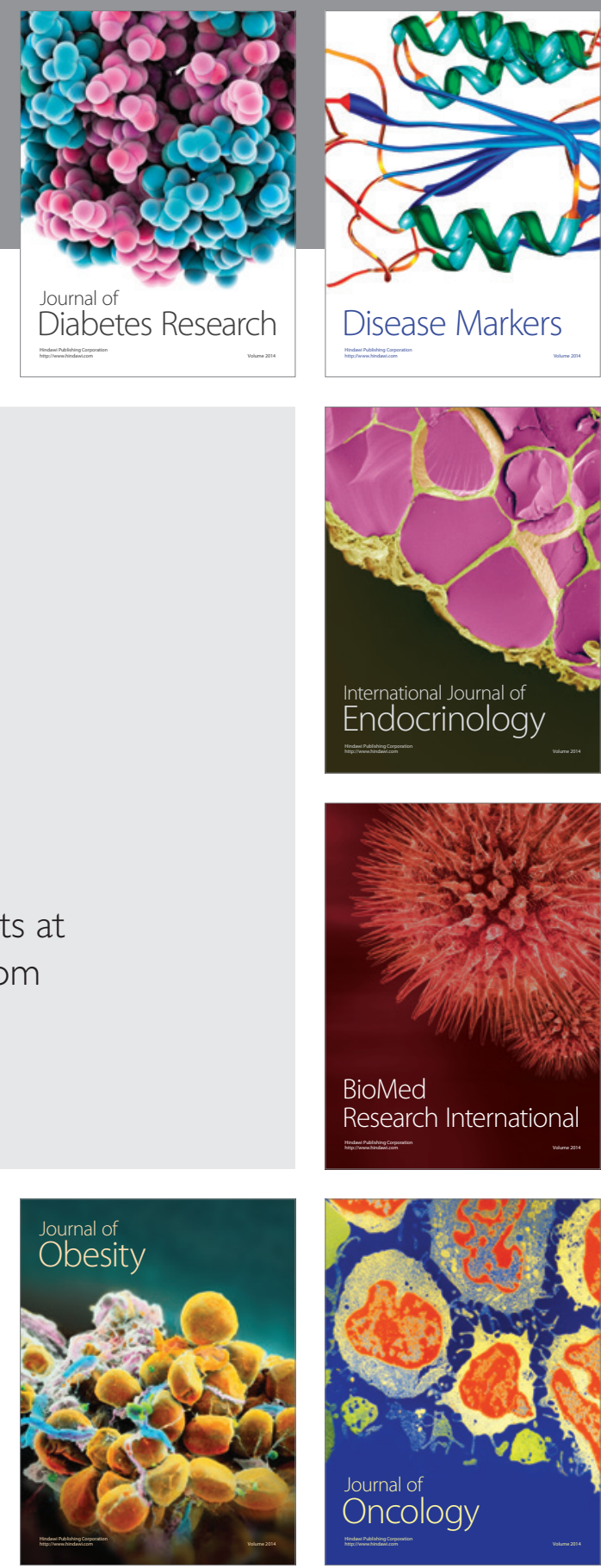

Disease Markers
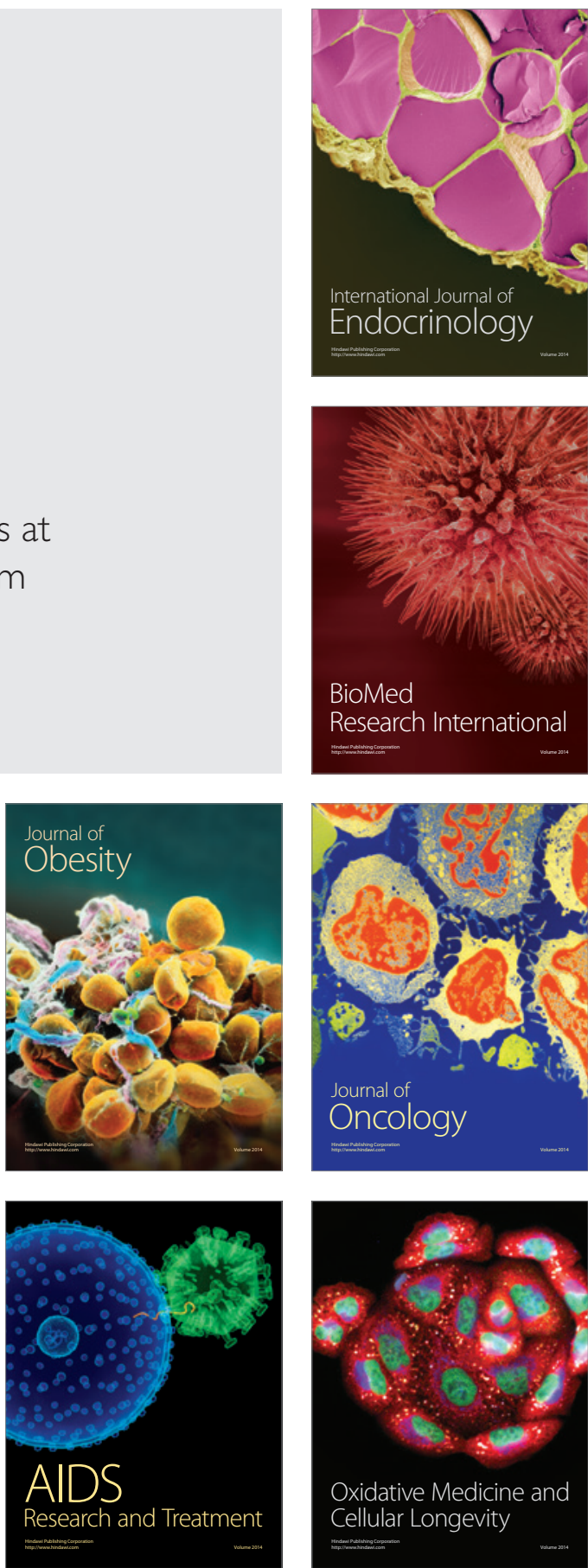